\title{
Effectiveness of a Vestibular Rehabilitation Protocol to Improve the Health-Related Quality of Life and Postural Balance in Patients with Vertigo
}

\author{
Heloísa Freiria Tsukamoto ${ }^{1}$ Viviane de Souza Pinho Costa ${ }^{2}$ Rubens Alexandre da Silva Junior ${ }^{2}$ \\ Gislaine Garcia Pelosi ${ }^{3} \quad$ Luciana Lozza de Moraes Marchiori ${ }^{2}$ Cláudia Regina Sanches Vaz ${ }^{4}$ \\ Karen Barros Parron Fernandes 2,5
}

\author{
${ }^{1}$ Department of Physical Therapy, Universidade Norte do Paraná \\ (UNOPAR), Centro Universitário Filadélfia, Londrina, Paraná, Brazil \\ 2 Biological and Health Sciences Research Center, Universidade Norte \\ do Paraná (UNOPAR), Londrina, Paraná, Brazil \\ ${ }^{3}$ Department of Physiological Sciences, Universidade Estadual de \\ Londrina (UEL), Londrina, Paraná, Brazil \\ ${ }^{4}$ Otorhinolaryngology Clinic, Londrina, Paraná, Brazil \\ ${ }^{5}$ School of Medicine, Pontifícia Universidade Católica do Paraná \\ (PUC-PR), Londrina, Paraná, Brazil
}

Int Arch Otorhinolaryngol 2015;19:238-247.
Address for correspondence Karen Barros Parron Fernandes, PhD, Centro de Pesquisa em Ciências da Saúde, Universidade Norte do Paraná, Rua Marselha, 591, Jd. Piza, Londrina, Paraná, 86041-140, Brazil (e-mail: karenparron@gmail.com).

\begin{abstract}
Introduction Dizziness can be characterized as a balance disorder that causes discomfort, leading to several functional limitations. Currently, vestibular rehabilitation has been highlighted as a possible treatment.

Objective Analyze the effects of completing a vestibular rehabilitation treatment protocol on quality of life and postural balance in patients with vestibular complaints, as well as to compare these effects between the patients taking or not taking antivertigo drugs.

Methods A nonrandomized controlled trial was performed with 20 patients previously diagnosed with vestibular diseases. Information regarding vertigo symptoms, quality of life as assessed through the Dizziness Handicap Inventory, visual analog scale of dizziness, and stabilometry using force platform was collected. Patients were treated for 12 weeks by a custom protocol. The sample was divided into two groups according to the use (medicated group, $n=9$ ) or not (control group, $n=11$ ) of antivertigo drugs. Results There was improvement in quality of life $(p<0.001)$ and intensity of dizziness

Keywords

- dizziness

- rehabilitation

- quality of life

- postural balance

- medicines $(p=0.003)$ with the intervention. An improvement of postural balance was observed through functional tests. However, no statistically significant difference was noted in stabilometry. When both groups were compared, no statistically significant differences between the variations of the variables analyzed were found in the re-evaluation session. Conclusion Quality of life and postural balance are improved with intervention. However, this improvement is not associated with pharmacologic treatment.
\end{abstract}

received

September 17, 2014

accepted

January 10, 2015

published online

May 6, 2015
DOI http://dx.doi.org/

10.1055/s-0035-1547523. ISSN 1809-9777.
Copyright $(2015$ by Thieme Publicações License terms

Ltda, Rio de Janeiro, Brazil
()(1) $\Theta \circledast$ 


\section{Introduction}

Defined as an illusion of movement of either the individual or his or her surrounding environment, dizziness can be characterized as a sensation of loss of balance in the body, due to the conflict of information between the sensory information originating in the vestibular, visual, and proprioceptive systems. ${ }^{1}$ Such a symptom can negatively influence the well-being of individuals of both genders in different ages. ${ }^{2,3}$

According to Ganança et $\mathrm{al}^{4}{ }^{4}$ dizziness is considered the second most prevalent symptom until the age of 65 years. After that age, it is the most common one, present in $80 \%$ of individuals over 75 years old. Hanley et al noted that the vertiginous syndromes represent 10.7 in every 1,000 medical consultations in the morbidity statistics for general practice in England. ${ }^{5}$ Neuhauser et al affirm that although these symptoms are more frequent in health primary care settings, they are often underestimated by professionals, who do not further investigate the symptoms. ${ }^{6}$

Together, discomfort, reduced motor capacity, and psychological suffering lead to functional limitations in personal care, tasks, and daily life activities, especially during the performance of tasks that require some postural balance, quick cephalic rotations, and good dynamic visual acuity. ${ }^{7-9}$ In such a context, frequent vestibular dysfunction affects life in family, professional, and social activities, causing losses in the individual's quality of life (QOL). ${ }^{10}$

Agus et al reported that there was still little agreement on the treatment and control of those symptoms. ${ }^{11}$ Depending on their etiology, vestibular dysfunctions may be treated with pharmacotherapy, physiotherapy, psychotherapy, and, rarely, surgery. ${ }^{12}$ For a long time, prescribing drugs for every person with vertiginous complaints, with no discrimination of the presented signs and symptoms, was common practice. Ganança et al stated that these monotherapeutic strategies may not be enough to solve vestibular complaints completely, in addition to predisposing the individual to the occurrence of side effects and addiction when use is prolonged. ${ }^{13}$

Currently, among the described forms of treatment to manage those dysfunctions, both in the acute and chronic stages, is a physiotherapeutic approach through vestibular rehabilitation (VR), ${ }^{5,7,14-19}$ which aims at reducing dizziness and body instability. VR is a low-cost and effective strategy in the treatment of vestibular disorder.

VR was first used in Brazil approximately two decades ago, with accounts of complete cure in $30 \%$ of cases and different levels of progress for $85 \%$ of the individuals. Therefore, according to Ricci et al, ${ }^{20}$ VR is not an etiologic treatment. Yet, these exercises allow new rearrangements of peripheral sensory information to happen., 41

Through neuroplasticity, this therapy aids in obtaining the so-called vestibular compensation, aiming at mitigation or elimination of unpleasant symptoms imposed by vestibular pathologies. Its proposed action is based on known mechanisms of adaptation, habituation, and substitution. Compensation is characterized by the sequence of plastic peripheral and central phenomena that occur after a vestibular lesion, with the purpose of re-establishing the system's function (i.e., the maintenance of the body balance). ${ }^{20}$

In the adaptation, the vestibular system learns to receive and process information-though distorted and incomplete-suiting it to the stimuli presented, promoting neural modifications. Habituation consists of a reduction of sensorial responses, due to the repetition of stimuli that, once becoming automatic, decreases vestibular response failures. Substitution occurs when sensory afferents are replaced. Through the visual and proprioceptive systems, information that is absent or that conflicts with the control of balance is substituted. ${ }^{22-24}$

VR attempts to improve the competence and well-being of the individual who has a vestibular pathology in the performance of daily life activities. It also aims at restoring spatial orientation as much as possible. It stimulates visual stabilization, reduces discomfort during head movements, and leads to greater stability in body posture, both when moving and while resting. ${ }^{9,25,26}$

Standardized VR protocols may not meet all functional necessities of patients with vestibular complaints. Thus, according to Morozetti et $\mathrm{al}^{9}{ }^{9}$ the combination of different therapeutic resources produces superior results, with less chance of recurrence. Therefore, to verify the contribution in the treatment of vestibular complaints, an adapted VR protocol was used in this study. This protocol involves activities aimed at assuring that the adaptation, habituation, and substitution mechanisms are facilitated, promoting the necessary neural plasticity for the individual's recovery.

In this context, the objectives to this study were: (1) analyzing the effectiveness in applying an adapted protocol for VR in QOL and postural balance of individuals who reported vestibular complaints, and (2) comparing these effects in individuals who were and who were not medicated with antivertigo drugs.

\section{Methods}

The study was characterized as a nonrandomized clinical study, developed within the facilities of the Physiotherapy Clinic in the City of Londrina, State of Paraná, Brazil, from March 2012 to December 2013. The research was approved by Ethics Committee for Research with Humans (report no. 177.276/12), and all participants signed an informed consent statement.

An intentional, convenient, and nonrandom sample was selected for the study, composed of individuals presenting with vestibular diseases who were referred to different medical services in the city of Londrina-PR, and who fall within the following eligibility criteria:

1. Inclusion criteria: individuals of both genders over 18 years old who presented with vestibular chronic dysfunction, dizziness or balance impairment, or other unspecific dizziness sensation for at least 3 months, who were clinically diagnosed by a medical doctor, and who presented vestibular symptoms scoring 1 point or over in the visual analog scale (VAS) to assess dizziness or scoring 16 
points or more in the Dizziness Handicap Inventory (DHI) questionnaire.

2. Exclusion criteria: individuals with severe visual and/or hearing impairment, orthopedic disorders that limited the performance of proposed activities, nervous system injuries that resulted in additional motor and/or sensitive damages, and/or peripheral vestibular diseases of the benign paroxysmal positional vertigo type or Meniere disease.

In total, 44 individuals who reported vestibular complaints were evaluated. Twenty of those participants did not complete the 12 proposed treatment sessions for several reasons: moving to another town/city, personal and/or family-related reasons, unavailability of time due to work, among other reasons. Thus, 24 individuals completed the VR protocol proposed in the study. However, two participants were excluded because they were absent more than three times and two other patients were excluded for using flunarizine, considering that its sedative effect could be a bias as it may impair the rehabilitation process. Finally, 20 participants constituted the two groups for the analysis: (1) the medicated group (MG), formed by individuals taking antivertiginous drugs (prescribed by doctors who referred them to physiotherapy service), and (2) control group (CG), formed by individuals who did not take antivertiginous drugs in the period they participated in the study (-Fig. 1).

\section{Study Proceedings}

The selected participants were initially evaluated with the following instruments:

- Participant's identification record, collecting personal data, anamnesis, data from complementary otological examinations, personal background, and main complaint.

- Evaluation record of vertigo symptoms, obtained through interview with the patient, with the objective of questioning individuals on the following variables: time of clinical evolution, characteristics, duration and periodicity of dizziness, and associated neurovegetativesymptoms. 22,25

- DHI questionnaire, to evaluate the self-perception of the disabling effects imposed by dizziness on QOL. The minimal score is 0 and the maximum possible is 100 points. The greater the score, the worse the vestibular patient's perception of QOL. Classification of the dysfunction was established by Yorke et al in the following way: slight dysfunction (16 to 34 points), moderate dysfunction (36 to 52 points), and severe dysfunction (over 54 points). ${ }^{26}$ Aquaroni Ricci et al reported that reduction in the total score of 18 or more points is considered a significant improvement in the QOL. ${ }^{27}$

- VAS to assess dizziness and to measure the intensity of vertigo symptoms, ranging in a numerical scale from 0 to 10 , in which 0 indicates the absence of dizziness and 10 the worst intensity of dizziness. ${ }^{28}$

- Timed Up and Go test (TUG), which evaluates risk of falling. Beginning at a seated position, the progress from sitting to

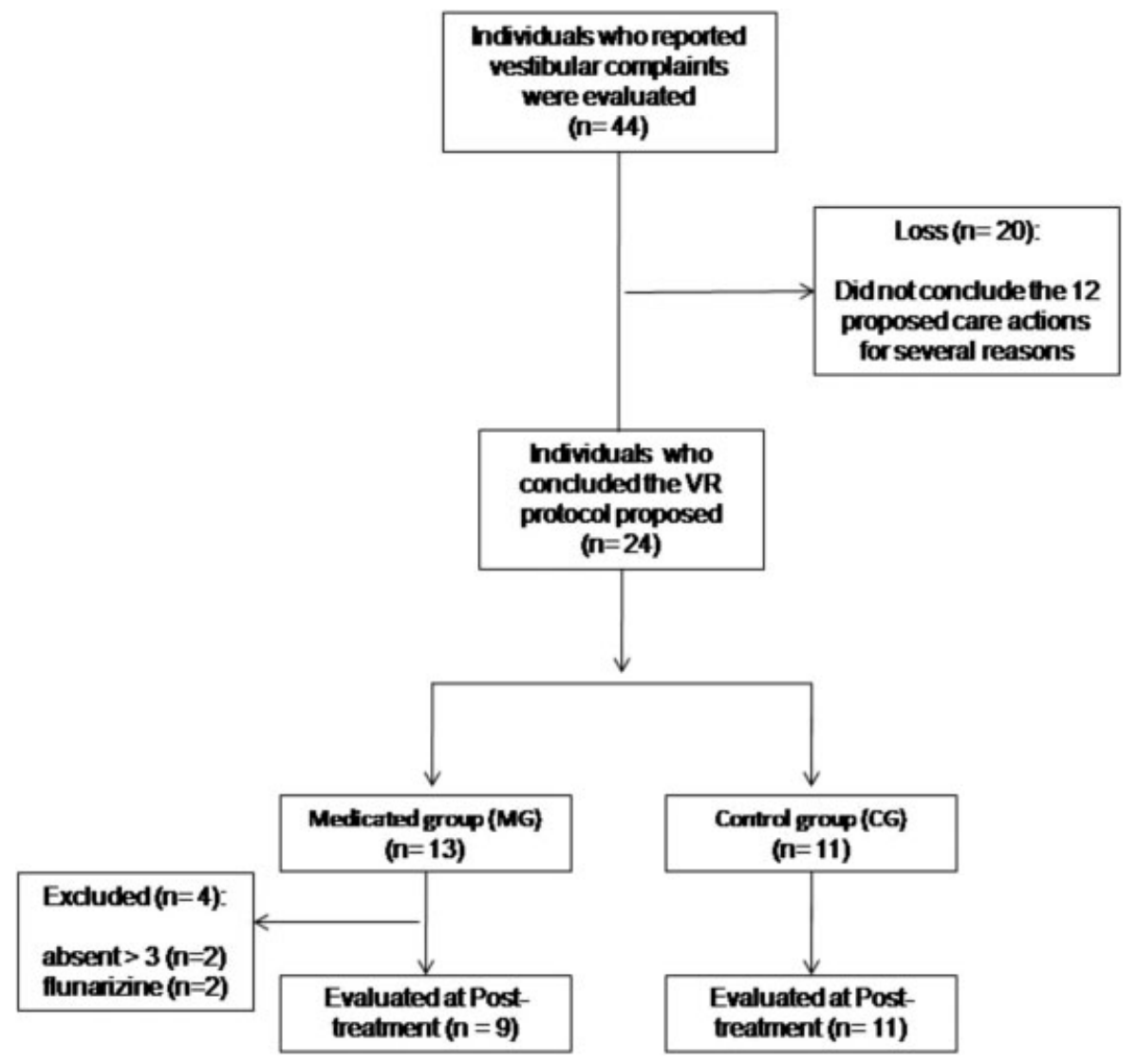

Fig. 1 Participants' selection flowchart. 
standing position is analyzed, including the stability and change of course, without making use of compensatory strategies. With the performance measured through the time (in seconds) necessary to take the test, the examiner asks the individual to stand up unassisted from a chair that he or she was totally supporting him- or herself and walk $3 \mathrm{~m}$, turn, return onto the same path, and sit again on the chair with his or her back upheld. The average from the three attempts in the test was adopted.

- Functional Reach test (FRT), a mobility test with emphasis on the evaluation of balance. The individual is asked to stand, sideward to a wall, with shoulder in 90-degree flexion and upper limbs in extension. Afterward, the individual is asked to make an anterior trunk flexion, so that his or her heels do not lose contact with the ground. Performance is measured by the distance of body displacement (in centimeters), considering the average of the three attempts in the test.

- Single leg stance test, to evaluate the static and functional balance, in which the individual is asked to stand on a single foot based on the ground (the side is chosen by the patient). Proof is considered negative when the individual can remain in this position for 30 seconds without changing the supporting base. If case proof is positive, the time in seconds that the individual could keep in the test position is recorded.

- Postural control, with a force platform named BIOMEC400 (EMG System do Brazil, SP Ltda.), with data sampled at 100 $\mathrm{Hz}^{29}$ The variables used were pressure center area (COP area, in $\mathrm{cm}^{2}$ ), average speed of COP (in $\mathrm{cm} / \mathrm{s}$ ), amplitude (in $\mathrm{cm}$ ) and average frequency of $\mathrm{COP}$ (in $\mathrm{Hz}$ ) in both motion directions: anteroposterior $(\mathrm{A} / \mathrm{P})$ and medial-lateral.

Participants were evaluated in the orthostatic position, in four distinct conditions: (1) bipedal support, with feet aligned, eyes open; (2) bipedal support, with feet aligned, with head rotation to the right and to the left, following audible feedback, using a metronome; (3) bipedal support, in semi-tandem position (right foot forward the left foot or the contrary, according to the participant's preference), eyes open (EC) and (4) eyes closed (EC). For each posture, the patient should remain on the force platform for 30 seconds. Two collecting procedures were made for tests 2 and 3, with a 30second interval between them. Only one attempt was made for test 1 . The order of postures was obtained by means of simple randomization (raffle), done by the patient him- or herself before the tests began.

The subjects were evaluated barefoot, with upper limbs relaxed beside the body, head positioned horizontally to the ground plane, eyes staring a target fixed on the wall at the same level of the eyes, in frontal distance of $2.5 \mathrm{~m}(8.2 \mathrm{ft})$ for tests with open eyes. ${ }^{29}$

\section{Intervention}

Patients were treated for 12 weeks, with more than one adapted protocol for VR, idealized by the authors of the study, based upon the following activities: cervical manual therapy exercises and shoulder girdle exercises, Cawthorne-Cooksey protocol exercises, and proprioceptive and balance training.
Guidelines were also given on the performance of daily home exercises to complement the proposed treatment.

Cervical manual therapy and shoulder girdle exercises were introduced due to the osteoarticular pathophysiological mechanisms of the upper cervical spine (as osteophytes and atlanto-occipital instability), which may cause dizziness as a result of cervical proprioception changes. ${ }^{25}$ It is important to highlight that the cervical region is one of the most important sources of proprioceptive information from the vestibular nuclei, ${ }^{30}$ and the introduction of exercises for this body segment aims at promoting vestibular adaptation in addition to muscle relaxation.

The cervical manual therapy and shoulder girdle therapy were applied in all treatment sessions, as an initial exercise. In the first four sessions of 30 minutes, activities were done in lateral decubitus and dorsal decubitus positions. Among the exercises were passive mobilization of the scapula; massage therapy in trapezius, rhomboids, scalenes, sternocleidomastoid, and pectoralis major muscles; passive stretching of neck and upper limb muscles; and pompages. From the fifth to the eighth session, manual therapy was reduced to 20 minutes. Form the ninth to the twelfth session, to 10 minutes.

The Cawthorne-Cooksey protocol consists of exercises that involve head movements, eye coordination tasks, overall body movements, and balance tasks, ${ }^{31}$ which are important to promote habituation and adaptation. These exercises were introduced in the adapted protocol and performed in the dorsal and lateral decubitus positions for the first treatment sessions and, posteriorly, in sitting position. In the final treatment sessions, these exercises were associated with the proprioception and balance activities in the orthostatic posture.

Finally, proprioceptive and balance trainings were performed in different environmental conditions as of the seventh session. These exercises involve substitution mechanisms, because in vestibular disorders, the normal abilities may be recovered through the increase in activities of other systems, such as the visual and proprioceptive systems. ${ }^{15}$ Therefore, to do these activities, therapeutic resources such as mats, foam of density 30, trampoline, equalizer, and square board were used, associating tasks with open and closed eyes, bipedal and one foot support, walking forward and in reverse.

Treatment sessions were individual, lasted 60 minutes once per week, and were performed for 12 consecutive weeks by a specialist professional physiotherapist.

\section{Reassessment}

At the end of the 12 sessions, patients were reassessed using the following evaluation tools: DHI questionnaire, VAS to assess dizziness, TUG, FRT, one-foot support test, and stabilometry.

Besides these tests, all patients answered the question: "Do you consider that today, at the end of 12 treatment sessions, your symptoms ..." to which three answers were possible: (1) "got better"; (2) "remained unchanged"; or (3) "got worse."

\section{Data Analysis}

Data were analyzed in a descriptive and analytical manner, making use of software programs Statistical Package for 
Social Sciences (SPSS, Chicago, IL, USA) version 20.0 and Microsoft Office Excel for Windows 2007(Microsoft Corp., Redmond, Washington, United States).

To verify normality of the data, the Shapiro Wilk test for the numerical variables was used. To analyze the differences between the parameters assessed before and after the intervention for the total sample, the Wilcoxon test was used, once the assumption of normality was not met.

The comparison between the groups was made by analysis of variation in data, measured in the initial and final assessments (variation $=$ final value - initial value). Once that was done, if the assumption of normality was not met for all varieties, the Mann-Whitney test was used. The chi-square test was used to verify the existence of association among the categorical variables.

Finally, to verify the effect size $(d)$, the following formula was used: $d=\left(x_{1}-x_{2}\right)$ /averages of the standard deviations (SDs), where $x_{1}$ is the average of the analyzed variable in the initial assessment, and $x_{2}$ is the average of the analyzed variable in the final assessment. The average to the SDs was calculated through the arithmetic mean of the standard deviations related to the initial and final assessment: $\left(\mathrm{SD}_{1}+\mathrm{SD}_{2}\right) / 2$. A confidence interval of $95 \%$ was established and significance level of $5 \%$ $(p<0.05)$ applied to all tests.

\section{Results}

Twenty participants completed the 12 study protocol treatment sessions, 19 women (95\%) and 1 man (5\%). Participants' average age was 59.6 years old (SD 13.9). The time of referred symptoms ranged from 3 to 36 years, with median of 3.5 (range 1.0 to 10.0 ) years. The other clinical and functional data are shown in - Table 1.

\section{Quality of Life}

There was a reduction in the DHI questionnaire values after the treatment, not only for the total but also for the threedomain score (physical, functional, and emotional), with a statistically significant difference ( - Table 2 ).

No participant had a score of 0 in the final assessment. However, there was a reduction the scores of 19 patients (95\%), with maintenance of score in 1 woman (5\%). Nevertheless, only 8 participants (40\%) obtained a reduction greater than 18 points. Four of those (20\%) initially had a disorder classified as severe. Thus, 8 individuals (40\%) changed from a moderate or severe condition to a better classification by the end of the intervention (-Table $\mathbf{3}$ ).

\section{Intensity of Dizziness}

A statistically significant difference was observed in the evaluation of the dizziness intensity, measured through VAS to assess dizziness before and after the intervention. In the initial assessment, 3 individuals (15\%) reported intense dizziness; 12 (60\%), moderate dizziness; and 5 (25\%), slight dizziness. In the reassessment, 5 individuals (25\%) presented score equals to 0 in the VAS to assess dizziness, and the 3 participants who initially reported intense dizziness showed an important reduction in values: 1 was reclassified as slight dysfunction and 1 as moderate dysfunction. - Table 2 shows a comparison of clinical and functional parameters evaluated before and after the intervention $(n=20)$.

\section{Physical and Functional Assessments}

There was a statistically significant difference in the assessment of the functional capacity favoring the intervention in the tests TUG, FRT, EO, and EC when compared with the values in the initial and final assessments (-Table 2).

\section{Postural Balance}

In the assessment of postural balance by analysis of COP, the residence time in the reassessment was 30 seconds for every test in all participants. One notes that the statistically significant differences favoring the intervention were found only for two analyzed parameters, especially regarding the movement A/P (-Table 4).

\section{Effect Size}

In the analysis of the effect size, the following values for the study variables were found: DHI total $(d=1.24)$; physical aspects $(d=1.34)$; functional aspects $(d=1.00)$; emotional aspects $(d=1.04)$; VAS to assess dizziness $(d=1.10)$; TUG $(d=0.31)$; FRT $(d=0.59)$; EO $(d=0.18)$; and EC $(d=0.52)$.

\section{Use of Antivertigo Medications}

Nine participants used antivertigo medications (group MG) under medical prescription, and the other 11 participants did not use any class of drugs for that purpose (group CG). The most commonly used medication was betahistine dihydrochloride by 8 participants (88.9\%).

\section{Comparison between Groups}

Finally, in the comparison between groups MG and CG at the end of the intervention, there was no statistically significant difference in the analysis of DHI, VAS to assess dizziness, the physicalfunctional tests of balance, and the stabilometry $(p>0.05)$.

There was no association between use of medication and the categorical variables of the study: main complaint $(p=0.86)$, period of crisis $(p=0.34)$, periodicity of crisis $(p=0.74)$, characterization of crisis ( $p=0.17)$, duration of crisis $(p=0.40)$, and associated neurovegetative symptoms ( $p=0.36)$.

When questioned on the symptoms after completing the 12 individual treatment procedures, 18 participants (90\%) reported improvement. Symptoms remained unchanged for 2 participants $(10 \%)$ after the treatment. Both participants belonged to the CG group.

\section{Discussion}

Analysis of the effects sizes showed that the negative impacts on the QOL and postural balance of individuals with a vestibular disease could be minimized with the proposed intervention. These data are in accordance to the study of Patatas et $\mathrm{al}^{23}$ who observed a significant improvement in the QOL (assessed with DHI) after an RV protocol, independent of age and gender. Morozetti et al also verified improvements in the QOL (measured with DHI) and in the intensity of 
Table 1 Baseline clinical data $(n=20)$

\begin{tabular}{|c|c|c|c|}
\hline Measure & Categories & Absolute frequency $(n)$ & Relative frequency (\%) \\
\hline \multirow[t]{2}{*}{ Gender } & Female & 19 & 95 \\
\hline & Male & 1 & 5 \\
\hline \multirow[t]{4}{*}{ Age $(y)$} & $20-40$ & 2 & 10 \\
\hline & $41-60$ & 7 & 35 \\
\hline & $61-80$ & 9 & 45 \\
\hline & $>80$ & 2 & 10 \\
\hline \multirow[t]{4}{*}{ Time of clinical evolution (y) } & $<1$ & 7 & 35 \\
\hline & $1-10$ & 10 & 50 \\
\hline & $10-20$ & 1 & 5 \\
\hline & $>20$ & 2 & 10 \\
\hline \multirow[t]{3}{*}{ Main complaint } & Dizziness & 10 & 50 \\
\hline & Dizziness and tinnitus & 4 & 20 \\
\hline & Dizziness and heavy head & 6 & 30 \\
\hline \multirow[t]{3}{*}{ Period of dizziness } & Anytime & 12 & 60 \\
\hline & Morning/afternoon & 5 & 25 \\
\hline & Evening/dawn & 3 & 15 \\
\hline \multirow[t]{3}{*}{ Periodicity of dizziness } & Daily & 12 & 60 \\
\hline & Weekly & 4 & 20 \\
\hline & Monthly/sporadic & 4 & 20 \\
\hline \multirow[t]{2}{*}{ Duration of dizziness } & Seconds/minutes & 9 & 45 \\
\hline & Hours/days & 11 & 55 \\
\hline \multirow[t]{4}{*}{ Characteristics of dizziness } & Unbalance & 7 & 35 \\
\hline & Vertigo & 5 & 25 \\
\hline & Unbalance and vertigo & 5 & 25 \\
\hline & Unbalance and obscured vision & 3 & 15 \\
\hline \multirow[t]{2}{*}{ Associated symptoms } & Yes & 19 & 95 \\
\hline & $\mathrm{No}$ & 1 & 5 \\
\hline
\end{tabular}

dizziness (assessed by dizziness VAS) in 20 individuals subjected to two different VR protocols. ${ }^{9}$ That same study highlighted that the group treated with a personalized protocol presented significant improvements in comparison with the group treated only with ocular exercises. ${ }^{9}$ These findings emphasize the importance of proposing a differentiated exercise protocol for the treatment of vestibular complaints, with activities that are complementary to those recommended by the standard protocols provided by literature, as was implemented in this research.

Ricci et al explained in their systematic review that VR helps in obtaining the vestibular compensation by means of central mechanisms of neuroplasticity, with the objective to soften or eliminate the vestibular symptoms. ${ }^{20}$ Therefore, VR has been considered a safe option once it does not present risks of side effects. Besides being cost-effective and efficient, ${ }^{18,21,27,32,33}$ it obtains positive results through training composed of exercises that stimulate repetitive movements of eye, head, and trunk, combined with conventional phys- iotherapeutic resources, such as cervical manual therapy, proprioception and balance exercises, and postural care.

Ganança et al reported that although there are several options to relieve or eliminate dizziness and its associated symptoms, the therapeutic regimen must be designed based on the individual's specific vestibular trouble, taking into account the elimination of the underlying diseases, control of vertigo and related neurovegetative and psychoaffective symptoms, improvement in the vestibular compensation, and prevention of the aggravating factors. ${ }^{13} \mathrm{~A}$ rapid onset of therapeutic action is essential for the patient's well-being, and the treatment must be well tolerated by the individuals, with a low incidence of adverse effects.

Regarding the ideal number of VR sessions, RossiIzquierdo et al reported improvement (using DHI and dynamic posturography) after five intervention sessions. ${ }^{34}$ Bayat et al also made use of DHI in the assessment of elderly individuals subjected to a VR protocol based on Cawthorne and Cooksey exercises for 2 months. ${ }^{35}$ They found that VR is an 
Table 2 Comparison of clinical and functional parameters evaluated before and after the intervention $(n=20)$

\begin{tabular}{|l|l|l|l|l|l|l|l|}
\hline \multirow{2}{*}{ Test } & \multicolumn{4}{l}{ Baseline } & \multicolumn{3}{l|}{ Posttreatment $(\mathbf{1 2}$ wk) } \\
\cline { 2 - 8 } & Median & $\mathbf{1}^{\circ} \mathbf{q}$ & $\mathbf{3}^{\circ} \mathbf{q}$ & Median & $\mathbf{1}^{\circ} \mathbf{q}$ & $\mathbf{3}^{\circ} \mathbf{q}$ & $\mathbf{p}$ \\
\hline DHI total & 41.0 & 27.0 & 58.5 & 17.0 & 10.5 & 37.0 & $<0.001^{\mathrm{a}}$ \\
\hline DHI physical aspects & 18.0 & 14.0 & 21.5 & 9.0 & 4.5 & 15.5 & $0.001^{\mathrm{a}}$ \\
\hline DHI functional aspects & 18.0 & 10.0 & 22.0 & 8.0 & 4.5 & 15.5 & $0.004^{\mathrm{a}}$ \\
\hline DHI emotional aspects & 10.0 & 4.0 & 17.0 & 4.0 & 0.5 & 7.5 & $0.001^{\mathrm{a}}$ \\
\hline VAS of dizziness & 4.0 & 2.00 & 6.00 & 1.0 & 0.25 & 3.0 & $0.003^{\mathrm{a}}$ \\
\hline TUG (s) & 6.45 & 5.85 & 7.57 & 5.68 & 5.34 & 6.53 & $0.010^{\mathrm{a}}$ \\
\hline FRT (cm) & 31.31 & 24.98 & 32.39 & 33.79 & 29.70 & 37.24 & $0.010^{\mathrm{a}}$ \\
\hline Single leg stance test EO (s) & 30.00 & 14.62 & 30.00 & 30.00 & 26.25 & 30.00 & $0.028^{\mathrm{a}}$ \\
\hline Single leg stance test EC (s) & 6.84 & 3.00 & 11.37 & 8.44 & 4.12 & 14.91 & $0.034^{\mathrm{a}}$ \\
\hline
\end{tabular}

Abbreviations: $1^{\circ} \mathrm{q}$, first quartile (25\%); $3^{\circ} \mathrm{q}$, third quartile (75\%); DHI, Dizziness Handicap Inventory; EC, eyes closed; EO, eyes open; FRT, Functional Reach test; TUG, Timed Up and Go test; VAS, visual analog scale.

${ }^{a} p<0.05$.

Table 3 Classification of vestibular dysfunction by DHI in baseline and posttreatment

\begin{tabular}{|l|l|l|l|l|}
\hline \multirow{2}{*}{ Baseline } & Posttreatment (12 wk) & Severe & Total \\
\cline { 2 - 6 } & Slight & Moderate & 0 & 7 \\
\hline Slight & 7 & 0 & 0 & 7 \\
\hline Moderate & 4 & 3 & 1 & 6 \\
\hline Severe & 4 & 1 & 1 & 20 \\
\hline Total & 15 & 4 & & \\
\hline
\end{tabular}

Abbreviation: DHI, Dizziness Handicap Inventory.

effective therapeutic method in the treatment of the elderly with chronic vestibular dysfunction. Considering that a greater period of intervention could increase the clinical results, in this study we chose 12 treatment sessions. However, a greater frequency of weekly sessions might increase the results obtained in the final assessment of the sample or might promote gains in a shorter time period of intervention.

Besides the benefits of VR on QOL, a positive impact on the results of the physical-functional assessments was also observed, in accordance with Giray et al and Jung et al. ${ }^{36,37}$ McGibbon et al reported the use of tai chi as a therapeutic option to increase the gain over the postural balance and, consequently, improve these individuals' QOL. ${ }^{38}$ Meldrum et al studied the use of virtual reality, with Nintendo Wii Fit Plus (USA) games, as a low-cost and playful intervention that may help the individuals' adherence to the proposed VR treatment. ${ }^{16}$ Aquaroni Ricci et al incremented Cawthorne and Cooksey's standardized protocol with activities that include exercise of flexibility, muscle strength, cognition, and sensorial interaction. ${ }^{27}$

However, in the analysis of postural control on the force platform, only six evaluated parameters presented statistically significant differences favoring the intervention. Still, some parameters had a final median greater than the initial value-for instance, the COP variable in the bipedal support with head rotation (BHR) test. At the end of the treatment, those individuals who presented greater amplitude in cervical rotation (effect of the manual cervical therapy and scapular waist) may have greater body displacement during the test on the force platform.

It is also convenient to mention that four different statistically significant forces were verified in the $\mathrm{A} / \mathrm{P}$ direction variable, which might have occurred because the proprioceptive and balance training exercises were performed mainly with body displacements in the A/P direction, such as walking forward and backward. So, special attention must be paid to exercises focusing lateral motions.

According to Horak, ${ }^{8}$ the most important role to postural control in the vestibular formation is the control of head and trunk orientation in space in relation to the gravitational force, because vestibular sensorial references are extremely important in the postural control for high frequencies and velocities of body motion. At the end of the study, among the conditions to the tests performed on the force platform-with the exception of bipedal support with head rotation-all involved the maintenance of an elastic position, which did little to assess the contribution of the vestibular system in the maintenance of the postural balance. Thus, the introduction of dynamic conditions in the assessment made on a force platform, involving situations of body movement or the exterior environment, may enable the verification of gains in vestibular compensation. 
Table 4 Comparison of stabilometric parameters evaluated before and after intervention $(n=20)$

\begin{tabular}{|c|c|c|c|c|c|c|c|}
\hline \multirow[t]{2}{*}{ Test } & \multicolumn{3}{|l|}{ Baseline } & \multicolumn{3}{|c|}{ Posttreatment (12 wk) } & \multirow[b]{2}{*}{$p$} \\
\hline & Median & $1^{\circ} \mathrm{q}$ & $3^{\circ} \mathrm{q}$ & Median & $1^{\circ} \mathrm{q}$ & $3^{\circ} \mathrm{q}$ & \\
\hline \multicolumn{8}{|l|}{ BEO } \\
\hline $\operatorname{COP}\left(\mathrm{cm}^{2}\right)$ & 1.30 & 0.80 & 3.83 & 1.21 & 0.91 & 2.10 & 0.41 \\
\hline VEL A/P (cm/s) & 0.83 & 0.64 & 1.20 & 0.76 & 0.66 & 0.95 & 0.36 \\
\hline VEL M/L (cm/s) & 0.61 & 0.50 & 0.75 & 0.58 & 0.51 & 0.72 & 0.51 \\
\hline AMP A/P $(\mathrm{cm})$ & 1.97 & 1.40 & 2.98 & 1.78 & 1.29 & 2.08 & $0.03^{\mathrm{a}}$ \\
\hline AMP M/L (cm) & 1.09 & 0.77 & 2.10 & 1.18 & 0.82 & 1.45 & 0.94 \\
\hline $\mathrm{FM} \mathrm{A} / \mathrm{P}(\mathrm{Hz})$ & 0.34 & 0.24 & 0.51 & 0.32 & 0.24 & 0.49 & 0.92 \\
\hline $\mathrm{FM} \mathrm{M} / \mathrm{L}(\mathrm{Hz})$ & 0.38 & 0.27 & 0.60 & 0.50 & 0.33 & 0.53 & 0.39 \\
\hline \multicolumn{8}{|l|}{ BHR } \\
\hline $\operatorname{COP}\left(\mathrm{cm}^{2}\right)$ & 2.90 & 1.73 & 5.47 & 3.10 & 1.70 & 4.60 & 0.85 \\
\hline VEL A/P (cm/s) & 1.16 & 0.96 & 1.49 & 1.10 & 0.91 & 1.32 & 0.26 \\
\hline VEL M/L (cm/s) & 0.90 & 0.69 & 1.13 & 0.86 & 0.75 & 0.98 & 0.52 \\
\hline AMP A/P $(\mathrm{cm})$ & 2.17 & 1.99 & 3.38 & 2.39 & 1.96 & 2.80 & 0.43 \\
\hline AMP M/L (cm) & 1.95 & 1.17 & 2.17 & 1.87 & 1.51 & 2.39 & 0.88 \\
\hline $\mathrm{FM} \mathrm{A} / \mathrm{P}(\mathrm{Hz})$ & 0.43 & 0.33 & 0.51 & 0.48 & 0.35 & 0.57 & 0.12 \\
\hline FM M/L (Hz) & 0.44 & 0.32 & 0.54 & 0.41 & 0.36 & 0.49 & 0.50 \\
\hline \multicolumn{8}{|l|}{ STEO } \\
\hline $\operatorname{COP}\left(\mathrm{cm}^{2}\right)$ & 4.24 & 3.11 & 7.13 & 3.07 & 2.21 & 5.42 & 0.13 \\
\hline VEL A/P (cm/s) & 1.43 & 1.19 & 1.84 & 1.31 & 1.14 & 1.52 & 0.76 \\
\hline VEL M/L (cm/s) & 1.26 & 1.14 & 1.48 & 1.22 & 1.11 & 1.37 & 0.95 \\
\hline AMP A/P $(\mathrm{cm})$ & 2.53 & 2.26 & 3.52 & 2.24 & 1.84 & 2.73 & $0.04^{\mathrm{a}}$ \\
\hline AMP M/L (cm) & 2.90 & 2.36 & 3.52 & 2.78 & 2.28 & 3.04 & 0.38 \\
\hline $\mathrm{FM} \mathrm{A} / \mathrm{P}(\mathrm{Hz})$ & 0.55 & 0.46 & 0.67 & 0.61 & 0.57 & 0.68 & 0.07 \\
\hline $\mathrm{FM} \mathrm{M} / \mathrm{L}(\mathrm{Hz})$ & 0.40 & 0.37 & 0.46 & 0.42 & 0.38 & 0.50 & 0.46 \\
\hline \multicolumn{8}{|l|}{ STEC } \\
\hline $\operatorname{COP}\left(\mathrm{cm}^{2}\right)$ & 6.99 & 3.80 & 13.10 & 4.99 & 3.69 & 8.16 & 0.23 \\
\hline VEL A/P $(\mathrm{cm} / \mathrm{s})$ & 2.00 & 1.62 & 2.80 & 1.79 & 1.51 & 2.58 & $>0.99$ \\
\hline VEL M/L (cm/s) & 1.80 & 1.54 & 2.52 & 1.88 & 1.70 & 2.38 & 0.21 \\
\hline AMP A/P $(\mathrm{cm})$ & 3.40 & 2.42 & 4.67 & 2.70 & 2.32 & 3.81 & 0.11 \\
\hline AMP M/L (cm) & 3.84 & 3.01 & 4.74 & 3.42 & 2.89 & 4.45 & 0.26 \\
\hline FM A/P (Hz) & 0.63 & 0.45 & 0.75 & 0.60 & 0.52 & 0.79 & 0.62 \\
\hline FM M/L (Hz) & 0.48 & 0.39 & 0.52 & 0.48 & 0.41 & 0.60 & 0.06 \\
\hline
\end{tabular}

Abbreviations: $1^{\circ} \mathrm{q}$, first quartile (25\%); $3^{\circ} \mathrm{q}$, third quartile (75\%); A/P, anteroposterior; AMP, amplitude; BEO, bipedal support with eyes open; BHR, bipedal support with head rotation; COP, pressure center area; FM, average frequency; M/L, medial-lateral; STEC, semi-tandem position with eyes closed; STEO, semi-tandem position with eyes open; VEL, average speed. ${ }^{a} p<0.05$.

Despite this fact, the residence time on the force platform was 30 seconds for all participants in all reassessment tests. This condition was not verified in the initial assessment. Still, the individuals provided positive reports in relation to the performance of tests, once they demonstrated they felt more comfortable to perform the tasks.

In this study, when groups MG and CG were compared, it was possible to notice the improvement in vestibular symptoms regardless of the pharmacological treatment with anti- vertigo drugs. According to Fabiani et al, ${ }^{39}$ antivertigo drugs must be reasonably used for the shortest time possible, due to the side effects arising from their prolonged use (i.e., the risk of Parkinsonism). One must be careful due to the interference these symptomatic drugs promote over the compensation that naturally occurs by the central nervous system, especially in vertigo cases that last for more than a couple of days. ${ }^{16}$

According to Santos et al, ${ }^{22}$ in their study with the elderly who complained about dizziness, the use of antivertigo drugs 
was not associated with a worse or better QOL in relation to the elderly who did not make use of them, probably because the pharmacotherapy did not have much effect in the chronic stage of uncompensated vestibular diseases. Therefore, to avoid additional harm to vestibular patients, it is necessary to perform field research that allows establishing strategies and protocols in the management of dizziness. ${ }^{40}$

One limitation of this study is the nonperformance of followup of participants during the posttreatment period to verify if the VR effects are maintained long term. Additionally, considering the diversity of the pharmacologic treatment used, it is not possible to establish a relation between the class, dose, or type of treatment in relation to the study variables.

Future studies might compare these results to the results of individuals who are subjected to a protocol with shorter intervention time or greater frequency of weekly sessions, aiming at establishing an ideal and sufficient number of treatment sessions. Still, it is important to assess individuals with vestibular diseases, using a distinct protocol on the force platform to propose dynamic activities to better analyze the role of the vestibular information in the control of body balance.

\section{Conclusion}

QOL and postural balance improve with intervention. Yet, such improvement depends on the pharmacologic treatment. The data have clinical applicability in the sense of stressing the importance of VR for the individual with vestibular disease. Finally, this technique must be applied by a specialized physiotherapist to promote elimination of the unpleasant symptoms related to this dysfunction.

\section{References}

1 Takano NA, Cavalli SS, Ganança MM, et al. Quality of life in elderly with dizziness. Braz J Otorhinolaryngol 2010;76(6):769-775

2 Bittar RS, Oiticica J, Bottino MA, Ganança FF, Dimitrov R. Population epidemiological study on the prevalence of dizziness in the city of São Paulo. Braz J Otorhinolaryngol 2013;79(6):688-698

3 Gazzola JM, Perracini MR, Ganança MM, Ganança FF. Functional balance associated factors in the elderly with chronic vestibular disorder. Braz J Otorhinolaryngol 2006;72(5):683-690

4 Ganança FF, Castro ASO, Branco FC, Natour J. Interferência da tontura na qualidade de vida de pacientes com síndrome vestibular periférica. Rev Bras Otorrinolaringol 2004;70(1):94-101 Available at: http://www.scielo.br/pdf/rboto/v70n1/a16v70n1.pdf

5 Hanley K, O'Dowd T, Considine N. A systematic review of vertigo in primary care. Br J Gen Pract 2001;51(469):666-671

6 Neuhauser HK, Radtke A, von Brevern M, Lezius F, Feldmann M, Lempert T. Burden of dizziness and vertigo in the community. Arch Intern Med 2008;168(19):2118-2124

7 Morettin M, Mariotto LD, Filho OAC. Evaluation of the effectiveness of rehabilitation vestibular in patients with vestibular dysfunction. Int Arch Otorhinolaryngol 2007;11(3):284-292 Available at: http:// www.internationalarchivesent.org/conteudo/pdfForl/441_eng.pdf

8 Horak FB. Postural compensation for vestibular loss and implications for rehabilitation. Restor Neurol Neurosci 2010;28(1):57-68

9 Morozetti PG, Ganança CF, Chiari BM. Comparison of different protocols for vestibular rehabilitation in patients with peripheral vestibular disorders. J Soc Bras Fonoaudiol 2011;23(1):44-50
10 Funabashi M, Santos-Pontelli TEG, Colafemina JF, Grossi DB. Proposta de avaliação fisioterapêutica para pacientes com distúrbio do equilíbrio postural. Fisioter Mov 2009;23(1):509-517 Available at: http://www2.pucpr.br/reol/pb/index.php/rfm?dd1=3386\&dd99= view\&dd98 $=\mathrm{pb}$

11 Agus S, Benecke H, Thum C, Strupp M. Clinical and demographic features of vertigo: findings from the REVERT registry. Front Neurol 2013;4:48

12 Singh KR, Singh M. Current perspectives in the pharmacotherapy of vertigo. Otorhinolaryngol Clin Int J 2012;4(2):81-85 Available at: http://www.jaypeejournals.com/eJournals/ShowText.aspx?ID $=3632 \&$ Type $=$ FREE $\&$ TYP $=$ TOP $\& I N=$ eJournals/images $/$ JPLOGO. gif\&IID=286\&isPDF $=$ YES

13 Ganança MM, Caovilla HH, Munhoz MSL, et al. Optimizing the pharmacological component of integrated balance therapy. Braz J Otorhinolaryngol 2007;73(1):12-18

14 Soto E, Vega R. Neuropharmacology of vestibular system disorders. Curr Neuropharmacol 2010;8(1):26-40

15 Marioni G, Fermo S, Zanon D, Broi N, Staffieri A. Early rehabilitation for unilateral peripheral vestibular disorders: a prospective, randomized investigation using computerized posturography. Eur Arch Otorhinolaryngol 2013;270(2):425-435

16 Meldrum D, Herdman S, Moloney R, et al. Effectiveness of conventional versus virtual reality based vestibular rehabilitation in the treatment of dizziness, gait and balance impairment in adults with unilateral peripheral vestibular loss: a randomised controlled trial. BMC Ear Nose Throat Disord 2012;12:3

17 Strupp M, Brandt T. Current treatment of vestibular, ocular motor disorders and nystagmus. Ther Adv Neurol Disord 2009;2(4): 223-239

18 Han BI, Song HS, Kim JS. Vestibular rehabilitation therapy: review of indications, mechanisms, and key exercises. J Clin Neurol 2011; 7(4):184-196

19 Moreira Bittar RS, Simoceli L, Bovino Pedalini ME, Bottino MA. The treatment of diseases related to balance disorders in the elderly and the effectiveness of vestibular rehabilitation. Braz J Otorhinolaryngol 2007;73(3):295-298

20 Ricci NA, Aratani MC, Doná F, Macedo C, Caovilla HH, Ganança FF. A systematic review about the effects of the vestibular rehabilitation in middle-age and older adults. Rev Bras Fisioter 2010;14(5): 361-371

21 Hillier SL, McDonnell M. Vestibular rehabilitation for unilateral peripheral vestibular dysfunction. Cochrane Database Syst Rev 2011;3(2):CD005397

22 Santos EM, Gazzola JM, Ganança CF, Caovilla HH, Ganança FF Impacto da tontura na qualidade de vida de idosos com vestibulopatia crônica. Pró-fono R Atual Cient 2010;22(4):427-432 Available at: http://www.scielo.br/pdf/pfono/v22n4/en_11.pdf

23 Patatas OHG, Ganança CF, Ganança FF. Quality of life of individuals submitted to vestibular rehabilitation. Braz J Otorhinolaryngol 2009;75(3):387-394

24 Tavares FdaS, Santos MF, Knobel KA. Vestibular rehabilitation in a university hospital. Braz J Otorhinolaryngol 2008;74(2):241-247

25 Gazzola JM, Ganança FF, Aratani MC, Perracini MR, Ganança MM. Clinical evaluation of elderly people with chronic vestibular disorder. Braz Otorhinolaryngol 2006;72(4):515-522

26 Yorke A, Ward I, Vora S, Combs S, Keller-Johnson T. Measurement characteristics and clinical utility of the Dizziness Handicap Inventory among individuals with vestibular disorders. Arch Phys Med Rehabil 2013;94(11):2313-2314 Available at: http:// www.archives-pmr.org/article/S0003-9993(13)00538-8/pdf

27 Aquaroni Ricci N, Aratani MC, Caovilla HH, Freitas Ganança F. Effects of conventional versus multimodal vestibular rehabilitation on functional capacity and balance control in older people with chronic dizziness from vestibular disorders: design of a randomized clinical trial. Trials 2012;13:246 
28 Bittar RS, Pedalini ME, Ramalho JO, Yoshimura R. Critical analysis of vestibular rehabilitation outcome according to dizziness etiology. Braz J Otorhinolaryngol 2007;73(6):760-764

29 da Silva RA, Bilodeau M, Parreira RB, Teixeira DC, Amorim CF. Agerelated differences in time-limit performance and force platformbased balance measures during one-leg stance. J Electromyogr Kinesiol 2013;23(3):634-639

30 Souza GS, Gonçalves DF, Pastre CM. Propriocepção cervical e equilíbrio: uma revisão. Fisioter Mov 2006;19(4):33-40 Available at: http://www2.pucpr.br/reol/public/7/archive/0007-00001813ARTIGO_4.PDF

31 Rogatto ARD, Pedroso L, Almeida SRM, Oberg TD. Proposta de um protocolo para reabilitação vestibular em vestibulopatias periféricas. Fisioter Mov 2010;23(1):83-91 Available at: http://www. scielo.br/pdf/fm/v23n1/08.pdf

32 Eleftheriadou A, Skalidi N, Velegrakis GA. Vestibular rehabilitation strategies and factors that affect the outcome. Eur Arch Otorhinolaryngol 2012;269(11):2309-2316

33 Rossi-Izquierdo M, Santos-Pérez S, Soto-Varela A. What is the most effective vestibular rehabilitation technique in patients with unilateral peripheral vestibular disorders? Eur Arch Otorhinolaryngol 2011;268(11):1569-1574

34 Rossi-Izquierdo M, Santos-Pérez S, Rubio-Rodríguez JP, et al. What is the optimal number of treatment sessions of vestibular rehabilitation? Eur Arch Otorhinolaryngol 2014;271(2): 275-280

35 Bayat A, Pourbakht A, Saki N, Zainun Z, Nikakhlagh S, Mirmomeni $\mathrm{G}$. Vestibular rehabilitation outcomes in the elderly with chronic vestibular dysfunction. Iran Red Crescent Med J 2012;14(11): 705-708

36 Giray M, Kirazli Y, Karapolat H, Celebisoy N, Bilgen C, Kirazli T. Short-term effects of vestibular rehabilitation in patients with chronic unilateral vestibular dysfunction: a randomized controlled study. Arch Phys Med Rehabil 2009;90(8): 1325-1331

37 Jung JY, Kim JS, Chung PS, Woo SH, Rhee CK. Effect of vestibular rehabilitation on dizziness in the elderly. Am J Otolaryngol 2009; 30(5):295-299

38 McGibbon CA, Krebs DE, Parker SW, Scarborough DM, Wayne PM, Wolf SL. Tai Chi and vestibular rehabilitation improve vestibulopathic gait via different neuromuscular mechanisms: preliminary report. BMC Neurol 2005;5(1):3

39 Fabiani G, Pastro PC, Froehner C. Parkinsonism and other movement disorders in outpatients in chronic use of cinnarizine and flunarizine. Arq Neuropsiquiatr 2004;62(3B):784-788

40 Betts T, Harris D, Gadd E. The effects of two anti-vertigo drugs (betahistine and prochlorperazine) on driving skills. $\mathrm{Br} \mathrm{J}$ Clin Pharmacol 1991;32(4):455-458 